\title{
Nanoparticle shape and configuration analysis by transmission electron tomography
}

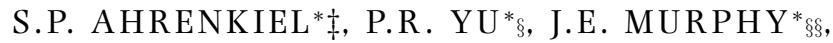 \\ J.M. NEDELJKOVIĆ $† \&$ B.S. DONOHOE* \\ *National Renewable Energy Laboratory, 1617 Cole Boulevard, Golden, CO 80401, U.S.A. \\ †Vinca Institute of Nuclear Sciences, P. O. Box 522, 11001 Belgrade, Serbia
}

Key words. Imaging, microscopy, nanoparticles, reconstruction, semiconductors, tomography.

\section{Summary}

Tomographic reconstruction by transmission electron microscopy is used to reveal three-dimensional nanoparticle shapes and the stacking configurations of nanoparticle ensembles. Reconstructions are generated from bright-field image tilt series, with a sample tilt range up to $\pm 70^{\circ}$, using single or dual tilt axes. We demonstrate the feasibility of this technique for the analysis of nanomaterials, using appropriate acquisition conditions. Tomography reveals both cubic and hexagonal close-packing configurations in multi-layered arrays of size-selected In nanospheres. By tomography and phase-contrast lattice imaging, we relate the three-dimensional shape of PbSe octahedral nanoparticles to the underlying crystal structure. We also confirm simplecubic packing in multi-layers of PbSe nanocubes and see evidence that the particle shapes have cubic symmetry. The shapes of $\mathrm{TiO}_{2}$ nanorod bundles are shown by tomographic reconstruction to resemble flattened ellipsoids.

\section{Introduction}

Transmission electron microscopy (TEM) is a premier method for the characterization of particle shapes and ordering in nanoparticle (NP) ensembles (Wang, 1998). High-resolution, phase-contrast lattice imaging provides crystallographic information of individual NPs in twodimensional (2D) projection. However, analysis of threedimensional (3D) particle shape from 2D projections is indirect. Inter-particle arrangements are usually revealed in relatively

Correspondence to: S. P. Ahrenkiel. Tel: 605394 5238; Fax: 605394 2365; e-mail: phil.Ahrenkiel@sdsmt.edu.

$\ddagger$ Present Address: South Dakota School of Mines and Technology, 501 E. Saint Joseph Street, Rapid City, SD 57701, U.S.A.

§Present Address: Optony Inc, Sunnyvale, CA 94086, USA.

$\S \S$ Present Address: Osram Syvania, Inc., Hawes Street, Towanda, PA 18848, U.S.A. low-magnification bright-field images and hollow-cone darkfield images, as well as small-angle electron-diffraction profiles. For each of these methods, however, a single datum yields only a 2D projection of the NP configurations; most 3D structural information arises by inference. TEM tomography provides a means to merge many individual 2D projection images into a single, 3D representation, which can be directly visualized and interpreted for micro-structural analysis.

Tomography is a natural extension of conventional TEM analysis, primarily requiring a relatively large sample tilt angle and sufficient computational power while also benefitting from microscope automation capability. Tomographic reconstruction requires each image of a tilt series to be an accurate structural projection (Weyland \& Midgley, 2004). Sophisticated software tools are available to accurately align large stacks of 2D images and generate their tomographic representations. A high tilt range favourably reduces the volume of the so-called missing wedge in the Fourier transform of the reconstruction, which causes an elongation normal to the projection plane (Midgley \& Weyland, 2003). By combining a pair of tilt series from orthogonal axes into a single reconstruction, the wedge is reduced to a smaller missing pyramid (Penczek et al., 1995; Mastronade, 1997; Weyland et al., 2001; Weyland \& Midgley, 2004; Arslan et al., 2006). Despite this improvement, surfaces parallel to the plane of projection remain substantially unrepresented in the reconstruction (Penczek \& Frank, 2006).

The bright-field imaging mode uses an objective aperture positioned around the direct beam in the back focal plane of the objective lens to generate amplitude (scattering) contrast (Reimer, 1997). Bright field is suitable for tilt-series tomography of amorphous materials and biological specimens, for which the scattering is predominantly incoherent, producing mass-thickness contrast, in the parlance of materials science. However, under bright-field conditions, crystalline materials also generate coherent scattering, when 
a region of the sample is oriented near the Bragg condition for a reflection, which causes abrupt variations in image intensity with tilt, known as diffraction contrast. Owing to a variety of factors, the diffraction contrast can even be evident when no objective aperture is used (Friedrich et al, 2005). Coherent diffraction contributes to the background of the reconstruction, reducing resolution, and rendering problematic the use of bright-field tomography for crystalline materials (Arslan et al., 2005). Thus, bright-field tomography is often assumed to be unfeasible for materials science. For an ensemble of randomly oriented nanocrystals, it is essentially impossible to avoid the Bragg condition through the range of a tilt series for all but a subset of the constituent NPs. Phase contrast can be enhanced by defocusing the objective lens, but this can also give rise to undesirable Fresnel fringes (Reimer, 1997; Weyland \& Midgley, 2004). Therefore, several groups pursuing tomography of nanocrystals have turned to annular dark-field imaging, using incoherent illumination, which is insensitive to Bragg diffraction (Yates et al., 2004; van Benthem et al., 2005a, b; Bals et al., 2006).

Unfortunately, direct access to instruments with both annular dark-field and high-tilt capability is limited. However, atomic resolution within a reconstruction is not required for much nanoscopic shape and configuration analysis. Brightfield tomography using conventional TEM can still provide useful information on structure, albeit with less than atomic resolution. The reconstructions can be correlated with lattice images, acquired separately. The diffraction contribution to bright-field image intensity also becomes less pronounced with decreasing crystallite size (Kaneko et al., 2005). This is encompassed in kinematical theory by a broadening in reciprocal space of the scattering potential's Fourier components (Reimer, 1997). Diffraction contrast is, therefore, a less significant problem for very small or defective crystalline NPs. Still, improvements in resolution using bright-field TEM tomography may become possible with the development of alternative reconstruction algorithms that minimize the deleterious effect of diffraction.

Studies of synthetic chemistry, fundamental physics and materials engineering can all benefit from improved 3D structural characterization of NP shapes and configurations. This information provides useful feedback for the refinement of growth conditions, the interpretation of experimental phenomena, and the utilization of the available properties for device applications. In support of these objectives, we demonstrate 3D analysis of various NP systems using TEM bright-field tomography. Acquisition was performed at relatively low magnification $(20000-40000 \times)$, with adherence to certain conditions: (1) a large objective aperture is used, (2) a small tilt increment and large tilt range are used and (3) the material under study is free-standing and not embedded in a strongly scattering matrix. The first condition minimizes diffraction contrast while preserving some massthickness contrast and resolution. The second condition is generally desirable to reduce gaps in the Fourier transform of the reconstruction. The third condition assures that positive contrast originates from the specimen at all orientations.

\section{Experiment}

All particles were prepared by colloidal chemistry. In spheres were produced from tri-tert-butylindium with trioctylphosphine (TOP) encapsulation (Nedeljković et al., 2004). PbSe octahedra were prepared from reaction of lead acetate, phenyl ether and acetic acid at $150^{\circ} \mathrm{C}$ in an argon atmosphere, with TOP and TOP-Se encapsulation. PbSe cubes were prepared by reaction of $\mathrm{PbO}$, oleic acid and 1-octadecene (Murphy et al., 2006). X-ray diffraction showed that both types of PbSe particles had the rocksalt structure (space group $F m \overline{3} m$ ). $\mathrm{TiO}_{2}$ nanorod bundles were synthesized by nonaqeous reaction between titanium chloride and benzyl alcohol (Abazović et al., 2006).

Samples were deposited from solution onto carbon-coated $\mathrm{Cu}$ TEM grids, typically 400-mesh, and then dried in vacuum. In some experiments, 10-nm diameter Au NPs were subsequently deposited on the grids for fiducial tracking. TEM tilt series were acquired on a Tecnai 20 Twin operated at $200 \mathrm{kV}$, using a high-tilt stage with range up to $\pm 70^{\circ}$. The tilt range was limited in some cases by the grid, rather than the holder, because regions of interest located near the grid bars often can obscure visibility at high tilt angle, due to the finite grid thickness (typically $50 \mu \mathrm{m}$ ). Tilt increments were specified as either a constant $1.0^{\circ}$ for the entire range, a value switched from $1.0^{\circ}$ within the range $\pm 45^{\circ}$ to $0.5^{\circ}$ beyond that range or a value determined by the Saxton scheme (Saxton \& Baumeister, 1984). Whenever possible, after first obtaining a tilt series about a single axis (A), a second tilt series was obtained about an orthogonal axis (B). A low-magnification map was obtained in the first orientation, with the region of interest highlighted. The sample was then removed from the holder and remounted with a rotation of approximately $90^{\circ}$. Using the map, the region of interest was located to obtain the B-axis tilt series.

Tomographic reconstructions were computed using the eTomo program, part of the IMOD 3.7 software package (Kremer et al., 1996). After coarse alignment of each tilt series by cross-correlation, a 3D model of the distribution of gold NPs, or other identifiable fiducial features, was generated and refined to track through the image stack and map the 2D image intensity onto the 3D reconstruction. When available, reconstructions from tilt series acquired on two roughly orthogonal axes were combined. For the combined reconstruction, each tilt series is computed separately, and a transformation between the two resulting reconstructions is established by fiducial or model matching. The Fourier components of the single-tilt tomograms are averaged in overlapping regions of reciprocal space; elsewhere the Fourier component is taken using the available value from one FFT, or 
as zero if the component falls within the remaining, unsampled pyramid of reciprocal space (Mastronade, 1997). Subsequent data manipulation was performed using Digital Micrograph 3.10 (Gatan, Pleasanton, CA, USA).

\section{Results}

This work demonstrates the feasibility of TEM brightfield tomography for relatively low-magnification structural analysis of nanomaterials. Despite the complications due to diffraction contrast, many groups routinely use tomography to reconstruct the 3D positions of crystalline NPs: Au fiducials, in particular, used for tracking in cellular biology. However, little materials-science research has examined by tomography the shapes and configurations of electronic materials and technologically important NPs. The bright-field approach is found to be adequate for nanoscopic shape and configuration analysis, in applications that do not require atomic resolution, which can be achieved separately.

\section{Stacking of In spheres}

In spheres serve as catalysts in the colloidal synthesis of NPs, particularly with nanorod and nanowire geometries, by solidliquid-solid reactions. The sphere size provides a means to affect the rod diameter. Like most spherical NPs, monolayers of size-selected nanospheres, deposited by slow evaporation of solvent on a surface, such as a carbon support film, naturally tend to condense into triangular lattices, which are readily identified by their projections in conventional TEM brightfield images. When the local coverage exceeds a monolayer, adjacent monolayers assemble into 3D arrays, but the contrast in projected images of these 3D, ordered regions becomes a superposition of contributions from multiple layers, greatly complicating the image interpretation (Fig. 1).

For example, single bright-field images of an ordered array of In spheres, taken with roughly $0^{\circ}$ tilt of the sample holder, show convoluted image contrast, which cannot be readily interpreted. In particular, the projection images do not provide direct information on the number of layers or their arrangement. Extraction of the 3D structure from a single exposure of such a region would require refinement of a structural model.

The full 3D packing arrangement of the nanospheres is revealed by tomographic reconstruction. Two distinct types of packing are represented in these truncated, three-layer stacks: (1) cubic-close packed (ccp) and (2) hexagonal close-packed (hcp). The ccp structure (space group $F m \overline{3} m$ ) has sequence ABCABC..., where $\mathrm{A}, \mathrm{B}$ and $\mathrm{C}$ represent three distinct, relative layer alignments. The hcp structure (space group $P 6_{3} / m m c$ ) has an ABAB... stacking sequence. These two packing arrangements and their differences are documented in numerous textbooks (e.g. Brandon \& Kaplan, 1999).

The determination of stacking arrangements is significant in that such self-assembly may be used to control optoelectronic

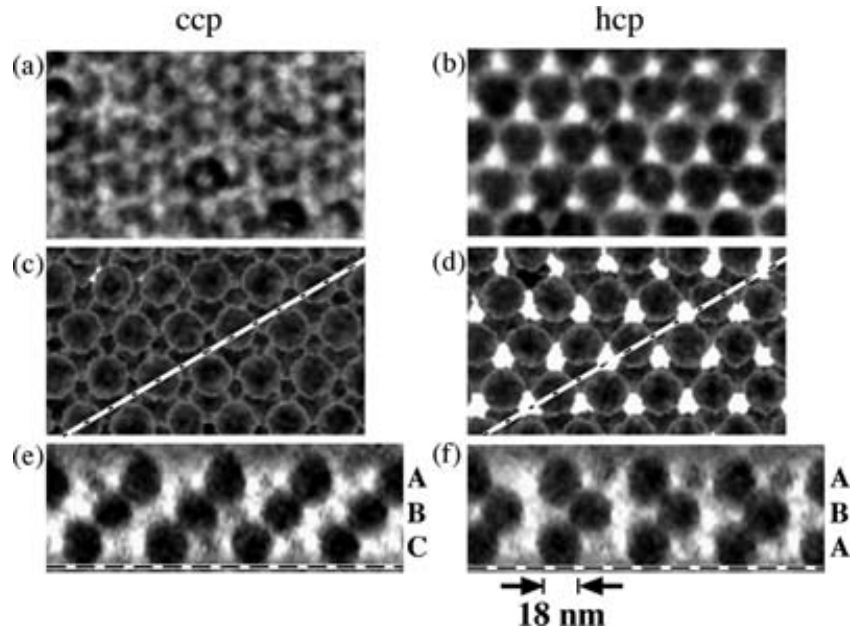

Fig. 1. Cubic close-packed (ccp) and hexagonal close-packed (hcp) stacking configurations among size-selected In nanospheres: (a, b) original, $\mathrm{O}^{\circ}$-tilt images; (c, d) superpositions of three tomographic slices; (e,f) cross-sectional slices taken along the diagonal, dotted lines shown in $\mathrm{c}$ and d. A, B, C refer to the lateral shift of each layer. Dashed lines indicate plane of contact with support film.

properties. The reconstruction is manipulated to view the layers in projection along directions oriented precisely perpendicular to the layers. Slices are then taken through the regions of highest density to more clearly reveal the relative positions of NPs within adjacent layers. Some definition of the particle shapes is lost due to the missing pyramid in reciprocal space and diffraction contrast, but does not impede the analysis of the particle arrangements. With sufficient refinement of the vertical structure, the arrays can be viewed in cross-section, which would be entirely impossible from a single projection image.

\section{PbSe Octahedra}

PbSe octahedral NPs having a six-pointed star shape provide an especially interesting application of TEM tomography (Fig. 2). Although the shapes are difficult to extract from individual TEM images, the tomographic reconstructions can be freely manipulated, providing direct shape analysis. Projections can be formed nearly precisely to the [100] (left), [110] (middle) and [111] (right) orientations. Phase-contrast latticeimages were used to assign crystallographic orientations to the observed shapes in projection. Observation along [100] gives a square projected shape; along [110] a roughly diamond shape is observed. Particles with 6-fold projected symmetry are also observed that do not give rise to resolvable lattice fringes.

It is evident that the six points are aligned with the $\langle 100\rangle$ axes of the underlying cubic structure. The points form at the intersections of the eight $\{111\}$ surfaces, which have slight concavity, accentuating the sharpness of the pointed tips. Slices parallel to the $\langle 100\rangle$ directions show 4 -fold symmetry. A slice 

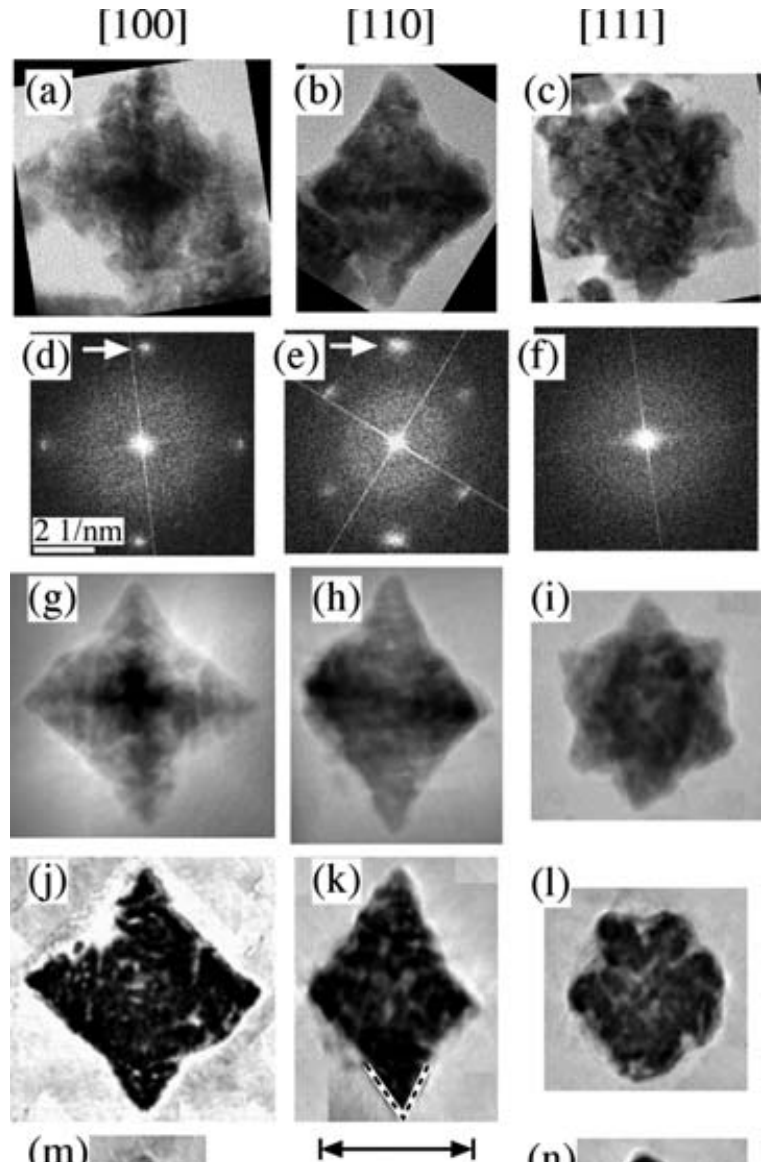

(m)

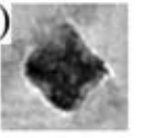

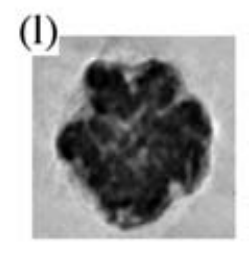

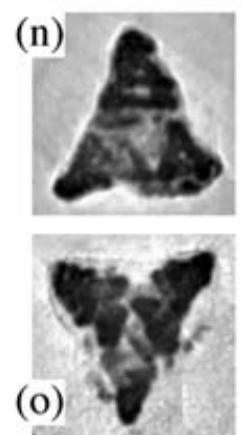

Fig. 2. PbSe octahedral NPs viewed in (left) [100], (middle) [110], and (right) [111] orientations: (a-c) phase-contrast lattice images; (d-f) Fourier transforms. Reconstruction of single NP: (g-i) full projections; (j-l) slices through particle centre; $(m)$ slice near tip, normal to tip axis; slices through (n) top face and (o) bottom face. Dotted lines highlight the projected tip angle.

through the particle centre shows a roughly square shape, with the concave, rounded edges normal to the $\langle 110\rangle$ directions. Slices through pointed tips, away from the centre, show that the particle tips are square in cross-section. The minimum angle subtended by the tips is observed in $\langle 110\rangle$ cross-section through the particle centre. Because of the concavity of the $\langle 111\rangle$ surfaces, the tips are sharper than those of an ideal octahedron. For example, the angle subtended along the tip

edges is measured to be only $72^{\circ}$, compared to $90^{\circ}$ for the regular octahedron, and between the opposing faces that form the tip, an angle of only $60^{\circ}$ is observed, compared to $70.5^{\circ}$ for planar faces. Slices normal to the $\langle 111\rangle$ directions vary dramatically depending on the distance of the slice from the particle centre. Slicing near opposite $\{111\}$ faces produces a triangular profile, with a reversal in orientation between the faces. Slicing through the particle centre produces a hexagonal shape.

Despite distortion of the surfaces with low inclination from the projection plane that results from the missing reciprocalspace pyramid, the symmetry of the octahedra allows for analysis along other orientations, for which the surfaces are more accurately represented. Referring to the cubic structure, for a [111]-oriented particle, three $\langle 100\rangle$ tips extend $54.7^{\circ}$ from the normal to the projection plane. This angle is less than the maximum tilt angle $\left( \pm 70^{\circ}\right)$ used, so the tips can be sectioned with relatively little distortion on (100). Similarly, three $\langle 110\rangle$ zones are present at $35.3^{\circ}$ from [111], allowing measurement of the minimum tip angle in a (110) section. Sections taken parallel to (111) are largely unaffected by the missing pyramid. Thus, the quoted tip-angle measurement is relatively insensitive to the limited tilt range.

Correspondence is also drawn between the projected profile of a particular particle in a single TEM image and the geometrical orientation of the particle on the support film. The [111] orientation places three neighbouring tips in contact with the carbon layer, which provides high configurational stability, by providing a wide base, with the centre-of-mass in close proximity to the surface.

\section{PbSe Cubes}

Tomography of PbSe nanocubes was pursued to examine the shapes and orientations of individual particles and 3D stacking arrangements in arrays. Analysis in conventional TEM projection images of low-density regions and single-layer arrays has shown roughly square 2D symmetry (plane point group $4 \mathrm{~mm}$ ) for the majority of these particles (Murphy et al., 2006). Lattice images reveal the [100] orientation of these particles along the direction corresponding to the square shape projection (Fig. 3).

The limited tilt range and strong crystallinity of these particles hinder an unambiguous global determination of particle shape by tomography. The [100] orientation presents a particular obstacle for a decisive shape determination of these NPs, because the Fourier components needed to generate the lateral facets are unrepresented in the reconstruction. Nonetheless, slices through the NPs extending normal to the plane of projection show roughly planar vertical faces, consistent with truncated cubic shapes (point group $m \overline{3} m$ ). For a particle inclined from the lateral plane, notice that the 4-fold symmetry of a vertical $\{100\}$ face is reconstructed adequately for identification. 

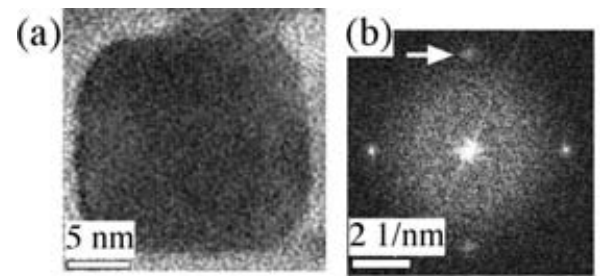

(c)

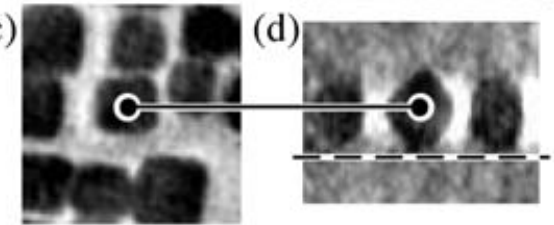

(e)

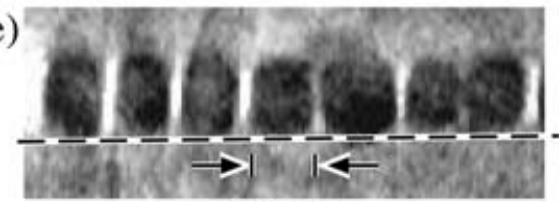

$18 \mathrm{~nm}$

Fig. 3. PbSe nanocube: (a) phase-contrast lattice image; (b) FFT showing [100] orientation. Reconstructions of PbSe nanocubes: A single NP inclined to the carbon support film is identified in (c) plan-view and (d) cross-section; (e) a row of several nanocubes, viewed in cross-section. Dashed lines indicate plane of contact with the support film. The arrow in (b) indicates the (020) periodicity.

Tomography also allows detailed inspection of the square, 2D ordering within individual layers, even those embedded within a dense 3D array. The individual layers are arranged predominantly in square lattices (plane group $\mathrm{pmm}$ ). By analysis of cross-sectional slices through 3D arrays, regions with simple-cubic ordering (space group $\operatorname{Pm} \overline{3} \mathrm{~m}$ ) are confirmed. Because of the high density of NPs in the array, the ordered stacking is most clearly visualized in $\langle 110\rangle$ orientations (Fig. 4).

\section{$\mathrm{TiO}_{2}$ Rod Bundles}

Bundles of $\mathrm{TiO}_{2}$ nanorods are well suited for BF tomography, because of the small, constituent crystalline domain sizes (Fig. 5), which reduces the orientation dependence for diffraction contrast, giving a less distorted representation of the overall particle shape. Lattice images show that these have the anatase structure and are oriented with [001] roughly parallel to a primary rod axis, which is around $23 \mathrm{~nm}$ in length. Each bundle typically contains several nanorods. The bundles have roughly oblate ellipsoidal shapes, with the long bundle axis tending to orient nearly parallel to the support film. The dimension perpendicular to the support film is of comparable length $(10 \mathrm{~nm})$ to the shorter, in-plane axis.

\section{Conclusions}

TEM tomography has many potential applications in materials nanoscience for the extraction of 3D structural information.
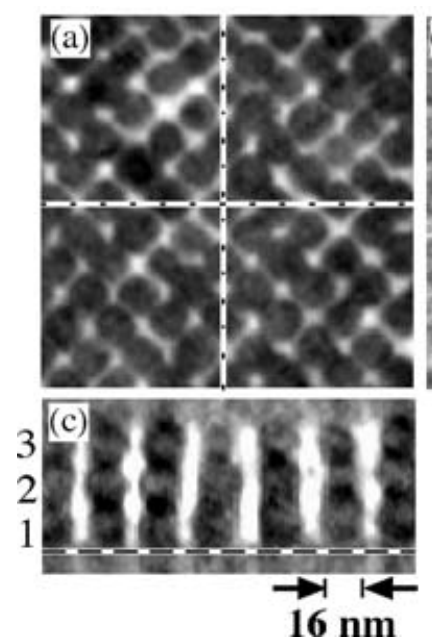

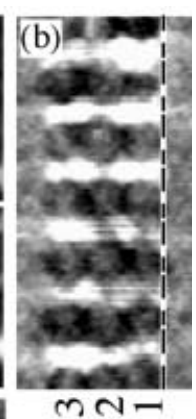

$\mathrm{N}=$
Fig. 4. Reconstruction of PbSe nanocubes in a simple-cubic array: (a) A plan-view slice through the bottom layer shows 2D square packing; (b, c) cross-sectional slices show that the region contains three adjacent layers, stacked in registry to form a 3D cubic array. Dotted lines indicate planes of sectioning. Dashed lines indicate plane of contact with the support film.
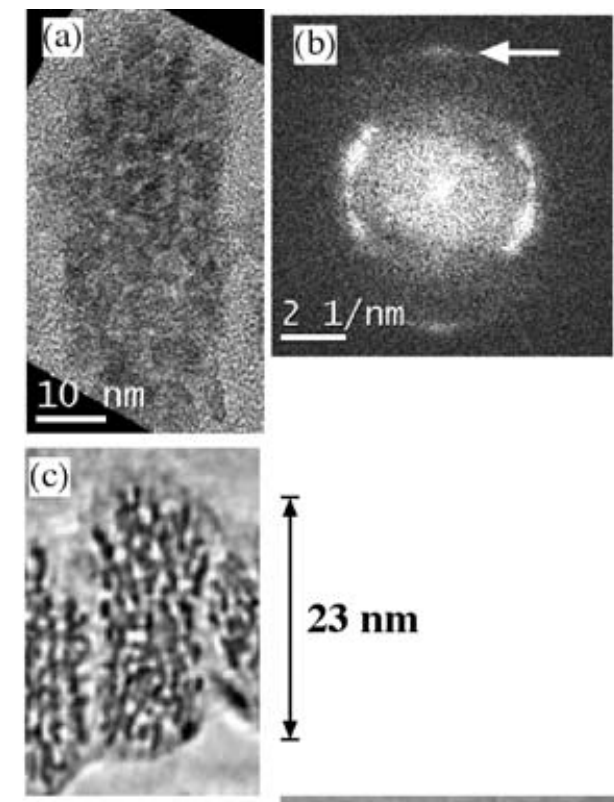

$23 \mathrm{~nm}$
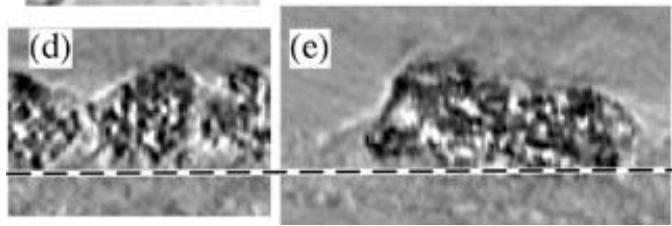

Fig. 5. $\mathrm{TiO}_{2}$ nanorod bundles: (a) Phase-contrast lattice image and (b) FFT. The arrow indicates the (004) periodicity. Reconstruction in (c) plan view and (d, e) cross-section. The dashed line indicates the plane of contact with the support film. 
Related studies have emphasized deficiencies in the use of bright-field imaging for tilt-series tomography (Friedrich et al., 2005). However, our preliminary work demonstrates that bright-field tomography is a viable method for the analysis of individual NP shapes and collective arrangements of NP ensembles. Although some questions in the nanoscopic regime can be readily addressed using the bright-field approach, we do anticipate that superior results could be achieved by using improved reconstruction methods and imaging techniques more advanced than those available for this work.

\section{References}

Abazović, N.D., Comor, M.I., Dramićanin, M.D., Jovanović, D.J., Ahrenkiel, S.P. \& Nedeljković, J.M. (2006) Photoluminescence of anatase and rutile $\mathrm{TiO}_{2}$ particles. J. Phys. Chem. B 110, 25366-25370.

Arslan, I., Tong, J.R. \& Midgley, P.A. (2006) Reducing the missing wedge: high-resolution dual axis tomography of inorganic materials. Ultramicroscopy 106, 994-1000.

Arslan, I., Yates, T.J.V., Browning, N.D. \& Midgley, P.A. (2005) Embedded nanostructures revealed in three dimensions. Science 309, 21952198.

Bals, S., Tendeloo, G.V. \& Kisielowski, C. (2006) A new approach for electron tomography: annular dark-field transmission electron microscopy. Adv. Mater. 18, 892-895.

Brandon, D. \& Kaplan, W.D. (1999) Microstructural Characterization of Materials. Wiley, New York.

Friedrich, H., McCartney, M.R. \& Buseck, P.R. (2005) Comparison of intensity distributions in tomograms from BF TEM, ADF STEM, HAADF STEM, and calculated tilt series. Ultramicroscopy 106, 18-27.

Kaneko, K., Moon, W.-J., Inoke, K. et al. (2005) Characterization of $\mathrm{TiO}_{2}$ - Ag nanocomposite particles prepared by spray pyrolosis process using transmission electron microscopy and three-dimensional electron tomography. Mater. Sci. Eng. A 403, 32-36.

Kremer, J.R., Mastronade, D.N. \& McIntosh, J.R. (1996) Computer visualization of three-dimensional image data using IMOD. J. Struct. Biol. 116, 71-76.
Mastronade, D.N. (1997) Dual-axis tomography: an approach with alignment methods that preserve resolution. J. Struct. Biol. 120, 343352.

Midgley, P.A.\&Weyland, M. (2003) 3D Electron microscopy in the physical sciences: the development of Z-contrast and EFTEM tomography. Ultramicroscopy 96, 413-431.

Murphy, J.E., Beard, M.C., Norman, A.G. et al. (2006) PbTe colloidal nanocrystals: synthesis, characterization, and multiple exciton generation. J. Am. Chem. Soc. 128, 3241-3247.

Nedeljković, J.M., Mićić, O.I., Ahrenkiel, S.P., Miedaner, A. \& Nozik, A.J. (2004) Growth of InP nanostructures via reaction of indium droplets with phosphide ions: synthesis of InP quantum rods and $\mathrm{InP}^{-\mathrm{TiO}_{2}}$ composites. J. Am. Chem. Soc. 126, 2632.

Penczek, P.A. \& Frank, J. (2006) Resolution in electron tomography. Electron Tomography, 2nd edn (ed. by J. Frank), pp. 307-330. SpringerVerlag, New York.

Penczek, P., Marko, M., Buttle, K. \& Frank, J. (1995) Double-tilt electron tomography. Ultramicroscopy 60, 393-410.

Reimer, L. (1997) Transmission Electron Microscopy: Physics of Image Formation and Microanalysis, 4th edn. Springer-Verlag, New York.

Saxton, W.O. \& Baumeister, W. (1984) Three-dimensional reconstruction of imperfect two-dimensional crystals. Ultramicroscopy 13, 57-70.

van Benthem, K., Lupini, A.R., Kim, M.J. et al. (2005a) Three-dimensional imaging of individual hafnium atoms inside a semiconductor device. Appl. Phys. Lett. 87, 034104.

van Benthem, K., Peng, Y. \& Pennycook, S.J. (2005b) Tomographic imaging of nanocrystals by aberration-corrected scanning transmission electron microscopy. Mater. Res. Soc. Symp. Proc. 839, 3-7.

Wang, Z.L. (1998) Structural analysis of self-assembling nanocrystal superlattices. Adv. Mater. 10, 13-30.

Weyland, M. \& Midgley, P.A. (2004) Electron tomography. Mater. Today 7, 32-40.

Weyland, M., Midgley, P.A. \& Thomas, J.M. (2001) Electron tomography of nanoparticle catalysts on porous substrates: a new technique based on Rutherford scattering. J. Phys. Chem. B 105, 7882-7886.

Yates, T.J.V., Laffont, L., Weyland, M., Zhi, D. \& Midgley, P.A. (2004) 3D analysis of semiconductor structures using HAADF STEM tomography. Inst. Phys. Conf. Ser. (Electron Microscopy and Analysis 2003) 179, 3134. 